\title{
Factors that may delay disappearance of trigeminal neuralgia after percutaneous balloon compression
}

\author{
Wenming $\mathrm{Lv}^{1,2}$, Wenjing $\mathrm{Hu}^{3}$, Lingyi $\mathrm{Chi}^{1}$, Liangwen $\mathrm{Zhang}^{4}$ \\ ${ }^{1}$ Department of Neurosurgery, Qilu Hospital, Cheeloo College of Medicine, Shandong University, Jinan, Shandong, China \\ ${ }^{2}$ Department of Neurosurgery, First People's Hospital of Ningyang County, Tai'an, Shandong, China \\ ${ }^{3}$ Shandong First Medical University and Shandong Academy of Medical Sciences, Tai'an, Shandong, China \\ ${ }^{4}$ Department of Neurosurgery, Shandong Provincial Hospital Affiliated to Shandong University, Jinan, Shandong, China
}

\begin{abstract}
Introduction. We set out to explore the factors that may affect delayed disappearance (DD) of trigeminal neuralgia (TN) after percutaneous balloon compression (PBC).

Material and methods. PBC was undergone by 221 patients with TN (95 male, 126 female) aged 33-89 years (mean 65). Delayed disappearance after surgery occurred in 59 patients. Follow-up continued until pain disappeared.

Results. A total of 221 patients, with an overall effective rate of $98.19 \%$, including 59 patients with DD (26.70\%), 158 patients with non-DD (71.49\%), and four patients without relief, were included in this study. The time of delayed disappearance ranged from two to 30 days after surgery, with an average of c.9 days. Factors related to delayed disappearance included symptom duration ( $\geq 8$ years), history of radiofrequency thermocoagulation, diabetes mellitus, herpes zoster, pain involving V2, and non-pear-shaped balloon. These were independent influencing factors $(p<0.05)$.

Conclusions. PBC is a safe and effective surgical method for treating TN. Delayed disappearance is a common phenomenon after surgery, and is influenced by many factors.
\end{abstract}

Key words: percutaneous balloon compression, trigeminal neuralgia, delayed disappearance, symptom duration

(Neurol Neurochir Pol 2022; 56 (2): 156-162)

\section{Introduction}

At present, the treatment methods for trigeminal neuralgia (TN) mainly include drug therapy, microvascular decompensation (MVD), radiofrequency thermocoagulation (RFT), and percutaneous balloon compression (PBC). $\mathrm{PBC}$ is mainly suitable for elderly patients with ineffective oral drugs, multi-branch pain, and poor surgical tolerance. It has the advantages of minimal trauma, high safety, and rapid effect.

However, pain may not disappear immediately after PBC surgery. Clinically, the phenomenon where pain does not disappear immediately after surgery, but gradually disappears during observation after surgery, is known as 'delayed disappearance' (DD). Nerve tissue undergoes sequential pathological changes after the ganglion is compressed by the balloon. When the filling pressure in the balloon is maintained for a period of time, most nerve cells are mechanically damaged. However, pathological changes do not occur, which may be the pathological basis of delayed disappearance. To date, no studies have reported on delayed disappearance in patients with $T N$ who underwent $P B C$ surgery.

Therefore, our study examined 59 patients with delayed disappearance of TN treated using PBC in our department. For the first time in this study we have examined related factors such as symptom duration, concomitant diseases, and balloon shape, to further explore the influencing factors of delayed disappearance, thus inferring the prognoses for patients and so guiding clinical work.

Address for correspondence: Liangwen Zhang, Shandong Provincial Hospital Affiliated to Shandong University, 324 Jingwu Weiqi Road, Jinan, 250021, Shandong Province, China; e-mail: zhangliangwen1221@163.com; co-corresponding author: Lingyi Chi

Received: 20.10.2021 Accepted: 4.01.2022 Early publication date: 14.02 .2022

This article is available in open access under Creative Common Attribution-Non-Commercial-No Derivatives 4.0 International (CC BY-NC-ND 4.0) license, allowing to download articles and share them with others as long as they credit the authors and the publisher, but without permission to change them in any way or use them commercially. 


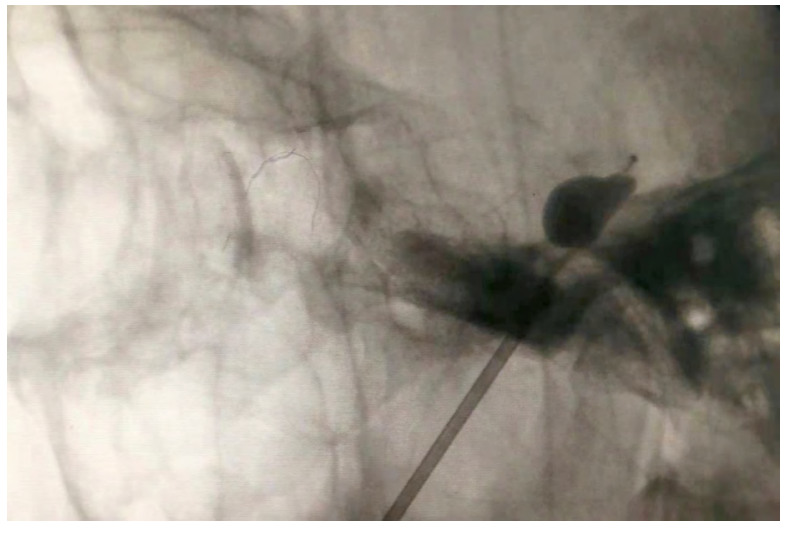

Figure 1. Pear-shaped balloon in situ. Pear-shaped balloon formed in Meckel's cave during percutaneous balloon compression (PBC) surgery

\section{Material and methods}

\section{Material}

A total of 221 patients who underwent $\mathrm{PBC}$ in our department from January 2018 to June 2021, and who were willing to be followed up after their surgery, were recruited as research participants. This study was approved and implemented by the Hospital Ethics Committee, and all patients who participated provided signed informed consent.

The inclusion criteria were: (1) medical history, symptoms and signs, and brain computed tomography and magnetic resonance imaging results that all met the International Headache Society (IHS)-II (2008) and IHS-III (2013) diagnostic criteria for TN $[1,2]$; and (2) oral carbamazepine invalid or serious adverse reactions.

Patients were excluded if: (1) they could not tolerate general anaesthesia, had dysfunction of the heart, lungs, liver, kidneys, or other vital organs, or had an obvious neurological impairment; or (2) had secondary TN; or (3) their medical records or follow-up information were incomplete.

\section{Methods}

Patients were placed in a supine position, and Hartel's puncture technique was performed after successful induction of general anaesthesia [3]. Under the fluoroscopic guidance of a C-arm machine (Ziehm 8000, Germany), the needle was inserted $3 \mathrm{~cm}$ outside the angle of incidence, and a No. 14 needle was inserted into the opening of the foramen ovale. A 4-F Fogarty catheter, connected to the T-joint, was slowly introduced into Meckel's cave along the puncture path. Next, $0.4-0.8 \mathrm{~mL}$ gadolinium diamine was injected to inflate the balloon to achieve a pear-like shape (Fig. 1). The compression continued for 3-5 min (average: $4 \mathrm{~min}$, Tab. 1). The contrast agent in the balloon was evacuated, and then the catheter and puncture needle were pulled out. The puncture port was compressed for $10 \mathrm{~min}$ before a sterile dressing was applied. Patients were followed up postoperatively via telephone or
Table 1. Compression time

\begin{tabular}{lc} 
Time $(\mathrm{min})$ & Number of patients (\%) \\
\hline 3 & $39(17.65)$ \\
4 & $143(64.70)$ \\
5 & $39(17.65)$
\end{tabular}

Variables are presented as $\mathrm{n}(\%)$ for nominal data; mean $\pm \mathrm{SD}$ or continuous data (average: $4 \mathrm{~min}$ ); $\mathrm{SD}$ - standard deviation

Table 2. Barrow Neurological Institute (BNI) pain intensity scale score

$\begin{array}{lr}\text { Score } & \text { Definition } \\ \text { I } & \text { No pain, no medication } \\ \text { II } & \text { Occasional pain, not requiring medication } \\ \text { III } & \text { Some pain, adequately controlled with medication } \\ \text { IV } & \text { Some pain, not adequately controlled with medication } \\ \text { V } & \text { Severe pain/no pain relief }\end{array}$

outpatient visits. This follow-up continued until the pain disappeared.

\section{Observation indices and effect evaluation}

(1) The time taken for pain symptoms to disappear, to be relieved, or to recur was recorded. The Barrow Neurological Institute (BNI) pain score was used to create an efficacy score (Tab. 2). "Immediate disappearance of pain" after surgery referred to a pain score of Grade I after the patient awoke from anaesthesia. "Delayed disappearance" (DD) was defined as when the patient's BNI score gradually changed to Grade I from the first postoperative day. "Non-improvement of postoperative pain" meant that the pain did not change, or was even aggravated, in the period between recovery from anaesthesia and the follow-up visit.

(2) The number of cases of intraoperative and postoperative complications (inhibitory reactions of the trigeminal nerve, facial numbness, facial hypoesthesia, masticatory-muscle weakness, tinnitus, diplopia, keratitis, herpes labialis, headache, cerebral haemorrhage, and blindness) was recorded.

(3) Any factors possibly related to delayed disappearance (including age, symptom duration, sex, side, history of RFT, MVD, concomitant disease, pain involving V2, balloon volume, compression time $[\leq 4 \mathrm{~min}]$, and shape) were evaluated.

\section{Statistical analysis}

Statistical Package for the Social Sciences (SPSS) 26.0 software was used for analysis, and the enumeration data was analysed using a $\chi^{2}$ test. Multivariate logistic regression analysis was performed on the basis of single-factor analysis to screen for independent risk factors and $\mathrm{p}<0.05$ was considered statistically significant. 


\section{Results}

\section{Demographic data and clinical characteristics}

A total of 221 patients with TN underwent PBC, performed by the same neurosurgeon in the Neurosurgery Department of Qilu Hospital, from January 2018 to June 2021. Table 3 shows the demographic data and clinical characteristics of the patients.

\section{Overall effect and complications of PBC for TN}

A total of 221 patients were analysed with an overall effective rate of $98.19 \%$, including 59 patients with delayed disappearance $(26.70 \%), 158$ patients with non-delayed disappearance (71.49\%), and four patients without relief (1.81\%) (Tab. 4). There were many complications during and after PBC. The incidence of inhibitory reaction of the trigeminal nerve during surgery was $97.3 \%$. The most common postoperative complications were: facial numbness, masticatory-muscle weakness, tinnitus, diplopia, and keratitis, which occurred at rates of $76.9 \%, 28.1 \%, 14.5 \%, 11.8 \%$, and $10.4 \%$, respectively (Tab. 5).

\section{Analysis of delayed disappearance time and influencing factors}

The duration of postoperative pain in the delayed disappearance group ranged from two to 30 days (Tab. 6), and the average duration of delayed disappearance was c. 9 days. In univariate logistic regression analysis, symptom duration ( $\geq$ 8 years), MVD, RFT, hypertension, diabetes mellitus, herpes zoster, pain involving V2, and non-pear-shaped balloon were all statistically significant $(\mathrm{p}<0.05)$ as set out in Tab. 7 . Multivariate logistic regression analysis showed that symptom duration ( $\geq 8$ years), RFT, diabetes mellitus, herpes zoster, pain involving $\mathrm{V} 2$, and non-pear-shaped balloon were independent factors for recurrence $(\mathrm{p}<0.05)$ as set out in Tab. 8.

\section{Discussion}

PBC surgery, an effective treatment for TN, is mainly suitable for elderly patients with ineffective oral drugs, multi-branch pain, and poor surgical tolerance. Percutaneous balloon compression (PBC) is based on the principle that the balloon selectively compresses the damaged myelinated nerve fibres that conduct pain in Meckel's cave, preserving the motor branches of the trigeminal nerve [4], and closing the trigger switch of the pain conduction pathway of the trigeminal nerve to eliminate pain. The statistical rates of immediate disappearance after $\mathrm{PBC}$ surgery in China and abroad vary significantly, ranging from approximately $74 \%$ to $100 \%[5-8]$. The incidence of delayed disappearance has been described as 9.31-19\%, which was different from our findings in the present study. However, the average duration of delayed disappearance was approximately nine days, which was consistent with the research by Li Zaiyu et al. [9] in China [5, 9, 10].
Table 3. Demographic characteristics of 221 patients

Sex

M

$\mathrm{F}$

126

Age (range; years) $65(33-89)$

$<40$

$2(0.9 \%)$

$41-50 \quad 12(5.4 \%)$

51-60 35(15.9\%)

$61-70 \quad 65(29.4 \%)$

$71-80 \quad 80(36.2 \%)$

$>80 \quad 27(12.2 \%)$

Symptom duration (range; years) $8(0.04-50)$

Distribution by side

Left

$79(35.7 \%)$

Right

$142(64.3 \%)$

Trigeminal division

$\mathrm{V}_{1}$

$4(1.8 \%)$

$V_{2}$

$76(34.4 \%)$

$V_{3}$

$12(5.4 \%)$

$V_{1+2}$

$57(25.8 \%)$

$V_{1+3} \quad 2(0.9 \%)$

$V_{2+3} \quad 40(18.1 \%)$

$\mathrm{V}_{1+2+3}$

$30(13.6 \%)$

Previous failed procedures

Microvascular decompression

Radiofrequency thermocoagulation

Percutaneous balloon compression

Glycerol gangliolysis

\section{Therapeutic effect of drugs}

No response to medical treatment

Multiple side effects of drugs

Concomitant disease

Hypertension

Diabetes mellitus

Coronary heart disease 25

Cerebral infarction 23

Herpes zoster 116

Chronic bronchitis 5

Sequelae of cerebral haemorrhage 2

Others (MS etc.)

Variables are presented as $\mathrm{n}(\%)$ for nominal data, $\mathrm{M}$ - male; $\mathrm{F}$ - female; $\mathrm{MS}$ - multiple sclerosis

Percutaneous balloon compression (PBC) is prone to causing complications such as inhibition of the trigeminal nerve reaction during surgery, postoperative facial numbness, 
Table 4. Barrow Neurological Institute (BNI) pain grades of 221 patients after percutaneous balloon compression (PBC)

\begin{tabular}{|c|c|c|c|}
\hline BNI pain grade & $\begin{array}{l}\text { Preoperatively of PBC, } \\
\text { number of patients (\%) }\end{array}$ & $\begin{array}{l}\text { Immediately after PBC, } \\
\text { number of patients (\%) }\end{array}$ & $\begin{array}{l}\text { During postoperative } \\
\text { period, number } \\
\text { of patients }(\%)\end{array}$ \\
\hline I & 0 & $158(71.5)$ & $217(98.2)$ \\
\hline II & 0 & $27(12.2)$ & 0 \\
\hline III & $21(9.5)$ & $28(12.7)$ & $1(0.4)$ \\
\hline IV & $65(29.4)$ & $8(3.6)$ & $3(1.4)$ \\
\hline V & $135(61.1)$ & 0 & 0 \\
\hline Total & $221(100)$ & $221(100)$ & $221(100)$ \\
\hline
\end{tabular}

This table reflects changes in numbers and percentages of pain in different grades before and after PBC. Variables are presented as $\mathrm{n}(\%)$ for nominal data

Table 5. Complications of 221 patients during/after percutaneous balloon compression (PBC) (n/\%)

\begin{tabular}{lc|}
\hline Complication & $\begin{array}{c}\text { Number of } \\
\text { patients (\%) }\end{array}$ \\
\hline Inhibitory reactions of trigeminal nerve & $215(97.3)$ \\
Cardiac arrest & $15(6.8)$ \\
Facial numbness & $17(76.9)$ \\
Severe facial hypoesthesia & $26(11.8)$ \\
Paresthesia & $14(6.3)$ \\
Masticatory-muscle weakness & $62(28.1)$ \\
Tinnitus & $32(14.5)$ \\
Diplopia & $26(11.8)$ \\
Keratitis & $23(10.4)$ \\
Labial herpes & $18(8.1)$ \\
Headache & $2(0.9)$ \\
Number and percentage of complications during or after PBC, variables are presented as $\mathrm{n}(\%)$ for \\
nominal data
\end{tabular}

paresthesia, and masticatory-muscle weakness. Because Meckel's cave, where the semilunar segment of the trigeminal nerve is located, is adjacent to the oculomotor, trochlear, abducens, and vestibular nerves and other structures, it is common for patients to experience diplopia, keratitis, tinnitus, and other complications due to excessive balloon filling. Most inhibitory reactions of the trigeminal nerve are transient, with an incidence as high as $97.3 \%$ in this study. The incidences of postoperative complications, such as facial numbness, paresthesia, and masticatory-muscle weakness, and their recovery times, which have been reported in many articles both in China and abroad, may be related to factors such as the size of Meckel's cave, the volume and shape of the balloon, and the compression time of the balloon $[11,12]$.

At present, there is a lack of research on factors influencing delayed disappearance after PBC surgery in China and abroad. Therefore, the focus of this study was to include many factors affecting delayed disappearance. Our study has confirmed for the first time in the world that symptom duration ( $\geq 8$ years), RFT, diabetes mellitus, herpes zoster, pain involving V2, and non-pear-shaped balloon are factors affecting delayed disappearance after PBC surgery.

It is surprising that symptom duration ( $\geq 8$ years) was a significant factor in our results. We believe that the longer the course of TN, the more obvious the nerve compression. After mechanical damage is caused by $\mathrm{PBC}$, it will take a period of time for the trigeminal nerve to adapt to the damage and for the neuroelectrophysiology to return to normal [5]. Yadav et al. [6] confirmed that the delay to cure in patients with facial spasm after surgery positively correlated with the length of the course of the disease, and even reported a formula to calculate that delay to cure. This would seem to demonstrate the delayed repair of cranial nerves after mechanical injury.

Radiofrequency thermocoagulation (RFT) utilises a difference in temperature tolerance between different nerve fibres of the trigeminal nerve to selectively destroy fine fibres that transmit pain while preserving the coarse fibres for transmitting touch, which have relatively high thermal resistance. Observation of nerve injury under the microscope after radiofrequency thermocoagulation is characterised by thermosetting degeneration, necrosis, fracture of the adventitia and nerve fibres, complete destruction of the myelin sheath and axonal structure of nerve fibres, and charring to varying degrees of connective tissues such as blood vessels $[13,14]$. At present, there is a lack of basic research on nerve fibre repair after treatment of TN with RFT. We speculate that the 'scar' after the repair of the adventitia and nerve fibres delays the conduction of electrical signals.

Diabetic peripheral nerve disease occurs in approximately two-thirds of patients with diabetes, and neuropathic pain is induced by sensory hyperalgesia due to increased intracellular glycated end products, increased activities of inflammatory cytokines and aldose reductase, oxidative stress, and other factors $[15,16]$.

Urban et al. conducted research on the involvement of the trigeminal nerve and facial nerve in patients with diabetes. They found that $60 \%$ of cases had distal symmetrical sensory nerve multiple lesions; using electrophysiological examination, they also found that a high-glucose environment would affect the function of the trigeminal nerve $[17,18]$. This indicates 
Table 6. Timetable of delayed pain disappearance (days)

\begin{tabular}{|c|c|c|c|c|c|}
\hline Number & Duration of DD & Number & Duration of DD & Number & Duration of DD \\
\hline 1 & 5 & 21 & 10 & 41 & 30 \\
\hline 2 & 9 & 22 & 7 & 42 & 2 \\
\hline 3 & 3 & 23 & 5 & 43 & 5 \\
\hline 4 & 13 & 24 & 21 & 44 & 5 \\
\hline 5 & 12 & 25 & 2 & 45 & 12 \\
\hline 6 & 20 & 26 & 5 & 46 & 21 \\
\hline 7 & 3 & 27 & 7 & 47 & 3 \\
\hline 8 & 7 & 28 & 7 & 48 & 6 \\
\hline 9 & 5 & 29 & 4 & 49 & 7 \\
\hline 10 & 3 & 30 & 10 & 50 & 7 \\
\hline 11 & 10 & 31 & 7 & 51 & 7 \\
\hline 12 & 12 & 32 & 21 & 52 & 10 \\
\hline 13 & 7 & 33 & 30 & 53 & 4 \\
\hline 14 & 30 & 34 & 10 & 54 & 12 \\
\hline 15 & 10 & 35 & 5 & 55 & 7 \\
\hline 16 & 3 & 36 & 6 & 56 & 2 \\
\hline 17 & 8 & 37 & 30 & 57 & 2 \\
\hline 18 & 5 & 38 & 3 & 58 & 14 \\
\hline 19 & 2 & 39 & 2 & 59 & 3 \\
\hline 20 & 3 & 40 & 21 & - & - \\
\hline
\end{tabular}

DD - delayed disappearance

Table 7. Univariate logistic regression analysis for delayed pain disappearance (DD)

\begin{tabular}{|c|c|c|c|}
\hline Variable & $\begin{array}{l}\text { DD } \\
(n / \%)\end{array}$ & $\begin{array}{c}\text { No DD } \\
(n / \%)\end{array}$ & $\mathbf{P}$ \\
\hline Age ( $\geq 65$ years) & $40(27.0)$ & $108(73.0)$ & 0.938 \\
\hline Sex: $M$ & $27(29.3)$ & $65(70.7)$ & 0.542 \\
\hline Symptom duration ( $\geq 8$ years) & $33(40.2)$ & $49(59.8)$ & 0.001 \\
\hline Microvascular decompression & $28(37.8)$ & $46(62.2)$ & 0.006 \\
\hline $\begin{array}{l}\text { Radiofrequency } \\
\text { thermocoagulation }\end{array}$ & $39(65.0)$ & $21(35.0)$ & 0.000 \\
\hline \multicolumn{4}{|l|}{ Concomitant disease } \\
\hline Hypertension & $32(40.5)$ & $47(59.5)$ & 0.001 \\
\hline Diabetes mellitus & $19(44.2)$ & $24(55.8)$ & 0.000 \\
\hline Cerebral infarction & $10(43.5)$ & $13(56.5)$ & 0.064 \\
\hline Coronary heart disease & $8(22.9)$ & $27(77.1)$ & 0.568 \\
\hline Herpes zoster & $58(50.0)$ & $58(50.0)$ & 0.000 \\
\hline Left side & $19(24.1)$ & $60(75.9)$ & 0.434 \\
\hline Involvement of V2 & $58(29.1)$ & $141(70.9)$ & 0.002 \\
\hline Balloon volume ( $\leq 0.4 \mathrm{ml})$ & $26(28.0)$ & $67(72.0)$ & 0.827 \\
\hline Compression time ( $\leq 4 \mathrm{~min})$ & $26(28.3)$ & $66(71.7)$ & 0.501 \\
\hline 'Non-pear-shaped' balloon & $36(72.0)$ & $14(28.0)$ & 0.000 \\
\hline
\end{tabular}

Statistically significant values are given in bold, and $p<0.05$ was considered a statistically significant difference
Table 8. Multivariate logistic regression analysis for delayed pain disappearance

\begin{tabular}{|c|c|c|c|}
\hline Variable & OR & $95 \% \mathrm{Cl}$ & $\mathbf{P}$ \\
\hline $\begin{array}{l}\text { Symptom duration ( } \geq 8 \\
\text { years) }\end{array}$ & 5.092 & $1.527-16.975$ & 0.008 \\
\hline $\begin{array}{l}\text { Microvascular } \\
\text { decompression }\end{array}$ & 0.220 & $0.051-1.760$ & 0.179 \\
\hline $\begin{array}{l}\text { Radiofrequency } \\
\text { thermocoagulation }\end{array}$ & 32.500 & $3.239-326.064$ & 0.003 \\
\hline Hypertension & 3.244 & $0.901-11.684$ & 0.072 \\
\hline Diabetes mellitus & 4.012 & $1.758-13.645$ & 0.048 \\
\hline Herpes zoster & 125.302 & $11.716-1,340.075$ & 0.000 \\
\hline Involvement of V2 & 29.069 & $2.112-400.058$ & 0.012 \\
\hline lon-near-shaned & 5170 & & \\
\hline
\end{tabular}

Statistically significant values are given in bold, and $p<0.05$ was considered a statistically significant difference; $\mathrm{OR}$ - odds ratio; $\mathrm{Cl}$ - confidence interval; $\mathrm{P}$ - probability; $\mathrm{V} 2$ - maxillary significant difference; $\mathrm{OR}-$ odds ratio; $\mathrm{Cl}-\mathrm{O}$
nerve (the 2 nd branch of trigeminal nerve)

that continuous cytokine stimulation in patients with diabetes is likely to be the influencing factor for the occurrence, aggravation, delayed disappearance, and recurrence of TN. At the same time, another result of our study (which has been received by 'World Neurosurgery') confirmed that there was a correlation between postoperative recurrence of $\mathrm{TN}$ and 
delayed disappearance, and that diabetes was a non-independent factor for recurrence.

Herpes zoster is neurotropic and can hide in the neurons of the posterior roots or ganglia of the nerves for years, or even decades. The reasons for delayed disappearance caused by the reactivation of herpes virus after $\mathrm{PBC}$ may be related to the following factors [19]: 1) the trigeminal nerve was damaged during surgery, and the irritation led to virus propagation; 2) the resistance and immunity of the patient decreased due to surgery, and the herpes virus in the semilunar ganglion of the trigeminal nerve was induced and activated; 3 ) the herpes zoster membrane ruptured to form an erosion or secondary suppurative infection, or even cause herpes zoster meningitis; and 4) surgical stimulation caused dissolution of chromatin in the trigeminal nerve ganglion cells, exudation of serous fluid under the stratum corneum, and generation of facial blisters. After antiviral treatment the pain symptoms of the patient resolve, along with the disappearance of herpes.

The expanded saccule directly and mechanically damaged the myelinated nerve fibres and nerves, and blocked the blood supply to the semilunar ganglion of the trigeminal nerve by pulling and pressing Meckel's cave, thus promoting fibre demyelination and pain conduction inhibition. There are more independent branches and anastomotic branches in the blood supply system of the maxillary branch (V2) of the trigeminal nerve than there are in V1 and V3 [20]. Therefore, in theory, patients with $\mathrm{TN}$ involving $\mathrm{V} 2$ are more likely to have delayed disappearance during PBC treatment.

A balloon that is pear-shaped is considered to be the key to the effectiveness and reduction of complications after PBC surgery $[21,22]$. The shape of the balloon is closely related to its location and insertion process. Poor location of a balloon can lead to a non-pear-shaped balloon morphology during surgery and influence the postoperative effect [23]. The compression effect of the non-pear-shaped balloon on the semilunar segment of the trigeminal nerve was worse than that of the pear-shaped balloon, and more recovery time was needed. This might be because of the insufficient effective contact area of balloon compression or the delay of vascular injury caused by mechanical traction.

\section{Future directions}

We have confirmed, for the first time, that symptom duration ( $\geq 8$ years), history of RFT, diabetes mellitus, herpes zoster, pain involving V2, and a non-pear-shaped balloon are independent influencing factors. This allows us to better judge the recovery time of patients after PBC surgery to verify the effect of the surgery and give patients a reasonable explanation of their condition.

\section{Limitations}

The sample size of this study was small, and other possible contributory factors (e.g. intra-balloon pressure, Meckel's cave size) were not analysed. There is a lack of basic research on delayed disappearance after PBC, and there was a lack of evidence to support hypotheses in our discussion.

\section{Conclusion}

Influenced by many factors, delayed disappearance is a common phenomenon after surgery. Because of the existence of delayed disappearance, we suggest waiting at least one month after surgery before judging whether $\mathrm{PBC}$ is invalid.

Acknowledgments: We are greatly indebted to all individuals who enrolled in our study as patients. This study was supported by 2020 Taian Science and Technology Innovation and Development Project (Policy Guidance) (2020NS168). We thank John Holmes, MSc, from LiwenBianji (Edanz) (www.liwenbianji.cn), for editing the English text of a draft of this manuscript.

Conflicts of interest: None.

Funding: None.

\section{References}

1. Headache Classification Subcommittee of the International Headache Society. The International Classification of Headache Disorders: 2nd edition. Cephalalgia. 2004; 24 Suppl 1: 9-160, doi: 10.1111/j.1468-2982.2003.00824.x, indexed in Pubmed: 14979299.

2. Headache Classification Committee of the International Headache Society (IHS). The International Classification of Headache Disorders, 3rd edition (beta version). Cephalalgia. 2013; 33(9): 629-808, doi: 10.1177/0333102413485658, indexed in Pubmed: 23771276.

3. He YQ, He S, Shen YX, et al. Clinical value of a self-designed training model for pinpointing and puncturing trigeminal ganglion. $\mathrm{Br} J$ Neurosurg. 2014; 28(2): 267-269, doi: 10.3109/02688697.2013.835379, indexed in Pubmed: 24628215.

4. Li F, Han S, Ma Yi, et al. Optimal duration of percutaneous microbalIoon compression for treatment of trigeminal nerve injury. Neural Regen Res. 2014; 9(2): 179-189, doi: 10.4103/1673-5374.125347, indexed in Pubmed: 25206799.

5. Deng Z, Liu R, Liu Y, et al. Factors That May Affect Delayed Relief Of Trigeminal Neuralgia After Microneurosurgery And The Long-Term Outcomes Associated With Delayed Relief. J Pain Res. 2019; 12: $2817-$ 2823, doi: 10.2147/JPR.S222467, indexed in Pubmed: 31632131.

6. Yadav S, Sonone RM, Jaiswara C, et al. Long-term Follow-up of Trigeminal Neuralgia Patients treated with Percutaneous Balloon Compression Technique: A Retrospective Analysis. J Contemp Dent Pract. 2016; 17(3): 263-266, doi: 10.5005/jp-journals-10024-1838, indexed in Pubmed: 27207209.

7. Mullan S, Lichtor T. Percutaneous microcompression of the trigeminal ganglion for trigeminal neuralgia. J Neurosurg. 1983; 59(6): $1007-$ 1012, doi: 10.3171/jns.1983.59.6.1007, indexed in Pubmed: 6631493.

8. Jain A. Comparative Analysis of Balloon Compression and Radiofrequency Ablation in Idiopathic Trigeminal Neuralgia: A Retrospective Study with a 24-Month Follow-up. Turk J Anaesthesiol Reanim. 2019; 47(2): 146-150, doi: 10.5152/TJAR.2019.53533, indexed in Pubmed: 31080957.

9. Li Z, Luo Y, Chen Y, et al. Analysis of influencing factors of complications related to micro-balloon compression for trigeminal neuralgia. Chin J Neurosur Dis Res. 2016; 15(2): 169-170. 
10. Sindou M, Leston J, Decullier E, et al. Microvascular decompression for primary trigeminal neuralgia: long-term effectiveness and prognostic factors in a series of 362 consecutive patients with clear-cut neurovascular conflicts who underwent pure decompression. J Neurosurg. 2007; 107(6): 1144-1153, doi: 10.3171/JNS-07/12/1144, indexed in Pubmed: 18077952.

11. Kouzounias $K$, Schechtmann $G$, Lind $G$, et al. Factors that influence outcome of percutaneous balloon compression in the treatment of trigeminal neuralgia. Neurosurgery. 2010; 67(4): 925-34; discussion 934, doi: 10.1227/NEU.0b013e3181eb5230, indexed in Pubmed: 20881557.

12. Asplund P, Linderoth B, Bergenheim AT. The predictive power of balloon shape and change of sensory functions on outcome of percutaneous balloon compression for trigeminal neuralgia. J Neurosurg. 2010; 113(3): 498-507, doi: 10.3171/2010.2.JNS091466, indexed in Pubmed: 20345223.

13. Fujihara F, Kim K, Kokubo R, et al. High-frequency thermal coagulation to treat middle cluneal nerve entrapment neuropathy. Acta Neurochir (Wien). 2021; 163(3): 823-828, doi: 10.1007/s00701-020-04404-8, indexed in Pubmed: 32415488.

14. Costa YM, Baad-Hansen L, Bonjardim LR, et al. Reliability of the nociceptive blink reflex evoked by electrical stimulation of the trigeminal nerve in humans. Clin Oral Investig. 2017; 21(8): 2453-2463, doi: 10.1007/s00784-016-2042-6, indexed in Pubmed: 28074292.

15. Todorovic SM. Is Diabetic Nerve Pain Caused by Dysregulated Ion Channels in Sensory Neurons? Diabetes. 2015; 64(12): 3987-3989, doi: 10.2337/dbi15-0006, indexed in Pubmed: 26604173.

16. Burand AJ, Stucky CL. Fabry disease pain: patient and preclinical parallels. Pain. 2021; 162(5): 1305-1321, doi: 10.1097/j. pain. 0000000000002152 , indexed in Pubmed: 33259456.
17. Urban PP, Forst $\mathrm{T}$, Lenfers $\mathrm{M}$, et al. Incidence of subclinical trigeminal and facial nerve involvement in diabetes mellitus. Electromyogr Clin Neurophysiol. 1999; 39(5): 267-272, indexed in Pubmed: 10421997.

18. Markiewicz MR, Callahan N, Miloro M. Management of Traumatic Trigeminal and Facial Nerve Injuries. Oral Maxillofac Surg Clin North Am. 2021; 33(3): 381-405, doi: 10.1016/j.coms.2021.04.009, indexed in Pubmed: 34116905.

19. Nair PA, Patel BC. Herpes Zoster. StatPearls [Internet] Treasure Island (FL) StatPearls Publishing. 2022 Jan, indexed in Pubmed: 28722854.

20. Li X, Yue J, Yang L, et al. Application of Antidromic Conduction Monitoring in Ganglion Radiofrequency Thermocoagulation for Locating Trigeminal Branches in Trigeminal Neuralgia. Pain Pract. 2016; 16(3): 305-310, doi: 10.1111/papr.12286, indexed in Pubmed: 25727990.

21. Chen JF, Tu PH, Lee ST. Repeated percutaneous balloon compression for recurrent trigeminal neuralgia: a long-term study. World Neurosurg. 2012; 77(2): 352-356, doi: 10.1016/j.wneu.2011.06.013, indexed in Pubmed: 22120364.

22. Asplund $P$, Linderoth $B$, Bergenheim AT. The predictive power of balloon shape and change of sensory functions on outcome of percutaneous balloon compression for trigeminal neuralgia. J Neurosurg. 2010; 113(3): 498-507, doi: 10.3171/2010.2.JNS091466, indexed in Pubmed: 20345223.

23. Wang Q, Chen C, Guo G, et al. A Prospective Study to Examine the Association of the Foramen Ovale Size with Intraluminal Pressure of Pear-Shaped Balloon in Percutaneous Balloon Compression for Trigeminal Neuralgia. Pain Ther. 2021; 10(2): 1439-1450, doi: 10.1007/ s40122-021-00311-7, indexed in Pubmed: 34460076. 